\title{
Control of a Remote Microscope Over the Internet
}

BioTechniques 22:1154-1157 (June 1997)

\section{Ron Maturo, Gary Kath, Reid Zeigler and Paul Meechan \\ Merck Research Laboratories, Rahway, NJ, USA}

\begin{abstract}
Globally connected research sites frequently find the need to share information on a timely basis. The sharing of data obtained from microscopy has historically required that the researcher take micrographs of the desired image and send the film to the other site or, more recently, scan the micrographs into a computer and send the micrographs through e-mail. The authors identified the need to control and view, in as close to real time as possible, images being viewed on a remote microscope. The goal was to develop a system that would be versatile, easy to learn and readily adapted from existing materials and that would allow several users to simultaneously view and control the microscope. The use of commercially available materials along with a simple, custom-designed slide holder allowed researchers at remote sites to view one of 15 slides and move the slide as needed. The penalty for use of the Internet vs. dedicated phone lines such as Integrated Services Digital Network (ISDN) is that only 1 frame/7 s can be viewed at video resolution. The advantages of cost and multiple, simultaneous use over a ubiquitous system outweigh the disadvantage for most users.
\end{abstract}

\section{INTRODUCTION}

While telemedicine is gaining greater acceptance (1) in the medical field, a literature search revealed that little has been published on the use of the Internet to control microscopes and transmit microscope images for basic research purposes $(2,3)$. This project was conceived as a method to help researchers to easily allow remote colleagues to view microscope images. The driving force behind this goal was the need to do collaborative science at sites throughout the world. For example, basic research is being conducted at a site in the United Kingdom with additional research occurring at a site in the United States. Prior methods for transmitting information required capturing the image on $35-\mathrm{mm}$ film, processing the film, scanning it into a computer, sending the digital images over an Integrated Services Digital Network (ISDN) line, printing the images and then viewing at the United States site. This process would take many hours, if not days, and involve many people. For this project to be cost-effective, the system needed to do the following: (i) run on a variety of computer platforms (Microsoft ${ }^{\circledR}$ Windows ${ }^{\circledR}$ and Windows NTTM $^{\mathrm{TM}}$ [Microsoft, Redmond, WA, USA], Macintosh ${ }^{\circledR}$ and UNIX [Apple Computer, Cupertino, CA, USA]); (ii) require little or no training; (iii) use off-the-shelf hardware and user software; (iv) be easy to adapt to existing microscopes; $(v)$ be capable of viewing by multiple users at one time; and (vi) be available to the entire scientific community within the Merck Research Laboratories (Rahway, NJ, USA) Intranet (use of the browser technology behind the company firewall). To satisfy the first three requirements, Netscape Navigator (Netscape, Mountain View, CA, USA) was chosen as the user interface. The Navigator runs on all required platforms and is supported by the computer resources department. There was a desire to use off-the-shelf hardware and software where possible to make this a cost-effective and easy way to add more microscopes to the Intranet as needed. The current system was developed using available equipment (cost if equipment is purchased is approximately $\$ 27000$ ). Additional software and one-time outside Perl programming added $\$ 7000$ to the project. The Intranet was chosen as the broadcast medium because it is a secure, cost-effective and well-maintained way to connect all of Merck's worldwide sites (10-Mbytes/s bandwidth currently maintained for the sum of all corporate traffic with T1 links available at some sites). Other communication methods were also considered, including ISDN. Although ISDN communication offers the ability to run over dedicated lines at transfer rates up to $128 \mathrm{kbytes} / \mathrm{s}$ per user, there are several disadvantages. First, a dedicated line would be needed between the microscope and each user's office. Second, the cost of the equipment (approximately $\$ 1000$ at each desk, excluding monthly charges) was too expensive for the users' needs. Third, the system would only be available to one user at a time.

To meet the needs of the various scientific staff members, the project was designed around three levels of control: $(i)$ Phase I of the project provided simple viewing of work being conducted on a remote scope, allowing scientists to simply watch the work while using a normal phone line to communicate with the scientists at the remote location; (ii) Phase II provided actual remote control of the microscope (x-axis/yaxis movement and autofocus) to allow remote scientists to move the $\mathrm{x}$-axis,y-axis stage; and (iii) Phase III provided for a 
slide "jukebox" for the selection of one of 15 slides for viewing, which allows scientists to view multiple images. This feature was important because the system is designed to work over multiple time zones, and in many cases, the system would be working without staff in attendance.

\section{MATERIALS AND METHODS}

\section{Phase I}

Early development of the project was begun on the Macintosh platform because many of the necessary components (i.e., Internet server software, video capture board) were already in place for conceptual testing. Since live streaming video is not practical because of network load, a delayed refresh was necessary. Using Timed Video Grabber software (written by Allon Stern; available at http://www.avernus. com/ allon/tvg.html) for timed video capture, we were able to successfully capture a frame of video from a color chargecoupled device (CCD) camera (Model DCX-960MD [Sony, Montvale, NJ, USA] or Model DEI-750) [Optronics Co., Goleta, CA, USA]) attached to a Leitz Laborlux 12 Microscope (Leica Imaging Systems, Cambridge, England, UK) and display it on a HyperText Markup Language (HTML) document within Navigator. The Sony camera has an optical resolution of 600 lines; however, the image ultimately is being displayed at the monitor screen resolution (typically $72 \mathrm{dpi}$ ). Experiments using 14 -inch monitors and VGA $(640 \times 480$ pixel resolution) suggested that the limit of resolution in this mode was on the order of 5 to $10 \mu \mathrm{m}$. SVGA $(1024 \times 768$ pixel resolution) monitor trials indicated the resolution was approximately a factor of two higher $(2.5-5 \mu \mathrm{m})$. Future enhancements to the system will allow for the capture and transmission of a high-quality image through file transfer protocol (FTP). The image was fed into a VideoVision ${ }^{\mathrm{TM}}$ capture board (Radius, Sunnyvale, CA, USA) at a user-defined rate. This image was then written as a joint pictures expert group (JPEG) file for delivery through an HTML document. Using the HTML Meta tag, the page was automatically updated every ten seconds. However, these initial conditions quickly pointed out several problems, including processor overload, user distraction and overall poor performance because the entire page had to redraw every time the screen was set to refresh. Subsequent testing showed that when using the setup at a local site, the refresh time could be as rapid as every four seconds. When the image was being sent overseas, the fastest acceptable refresh attainable was every seven seconds. This 4-7-s refresh rate was found to be acceptable, as the user needed time to view the image on the screen. While the initial testing was underway, Netscape released Navigator 2.0, which introduced two useful software changes for this project: the development of the Frame tag within Navigator and the support for plug-in technology. This helped to solve the problem of the entire page redrawing and the potential problem in Phase II of how to provide a graphical interface for the control of the microscope.

At this point, we moved the project over to a Microsoft Windows NT Version 3.51 Workstation (later upgraded to
Microsoft NT 3.51 Server software) environment for further development. This shift was made because the majority of image analysis systems within Merck are Microsoft Windowsbased. In addition, our choice of Purveyor ${ }^{\mathrm{TM}}$ Internet server software (Process Software, Framingham, MA, USA) required Microsoft Windows NT Version 3.51. A PC was constructed, containing a Pentium ${ }^{\circledR} 133 \mathrm{MHz}$ processor, 2-Gbyte hard drive, 64-Mbyte random access memory (RAM) and a $6 \times$ CD-ROM.

With the addition of the Frame tag within the HTML document, a page was created in which only the microscope image refreshed every seven seconds. The HTML document consists of two different documents, the top image consisting of the company-required corporate logo and the bottom image showing the micrograph. With the introduction of Navigator Version 3.0, the Frame tag was modified to not show the frame borders, so that the users are given the impression of working on a single page. The micrograph image was still redrawn using the Meta command inside of Navigator, but because of the frames, only a portion of the user's screen was redrawn (Figure 1). A Pause button has been provided that stops the screen from redrawing so that the viewer can more closely examine the image on the screen. If the image is of interest, it can be saved to the user's computer simply by using the Navigator command to save the image to disk.

With all the HTML documents created, we needed to address the issue of a timed video capture on a Microsoft Windows NT platform. After several unsuccessful attempts at creating the necessary software and observing a reduced image detail using a video board within Microsoft Windows NT, we decided to leave the video capture responsibilities on a Macintosh computer that would write the JPEG file directly to the Intranet server. This move also prevented processor overload on the Microsoft Windows NT system.

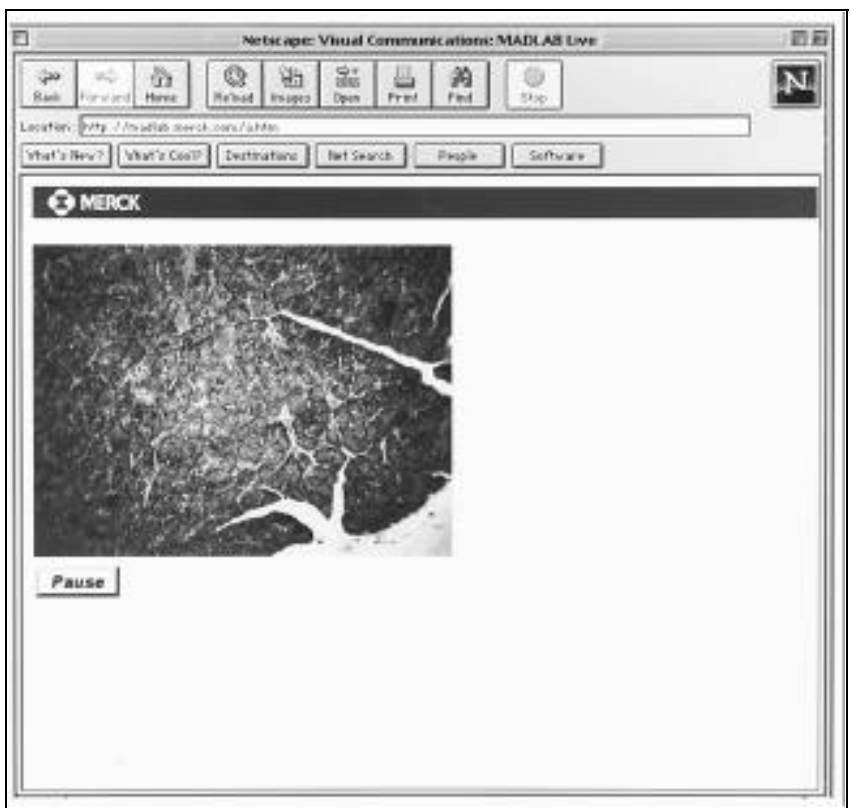

Figure 1. Screen capture of Phase I HTML page viewed through Navigator Version 3.0. The page shows the image, which is refreshed every seven seconds, and a non-refreshed logo and Pause button. 


\section{Phase II}

To fully develop Phase II, minimum computer standards were established. The user must run Netscape Navigator Version 2.02 or later, containing the Shockwave plug-in (Macromedia, San Francisco, CA, USA) and is suggested to have a 17-inch or larger monitor. We provided all the necessary software upgrades and plug-ins for downloading directly from the microscope Intranet site. The browser is available within the facility through a corporate site license with Netscape for new users and upgrades, while the Shockwave plug-in is freeware.

Using the same Leitz microscope with a motorized stage (Model MAC 2000; Ludl, Hawthorne, NY, USA) (Figure 2), the requirements for the controls were laid out. Rather than limit the user to preset steps, we determined that the interface had to be interactive. An interface was developed that provides for unlimited positioning of the stage, one-step return of

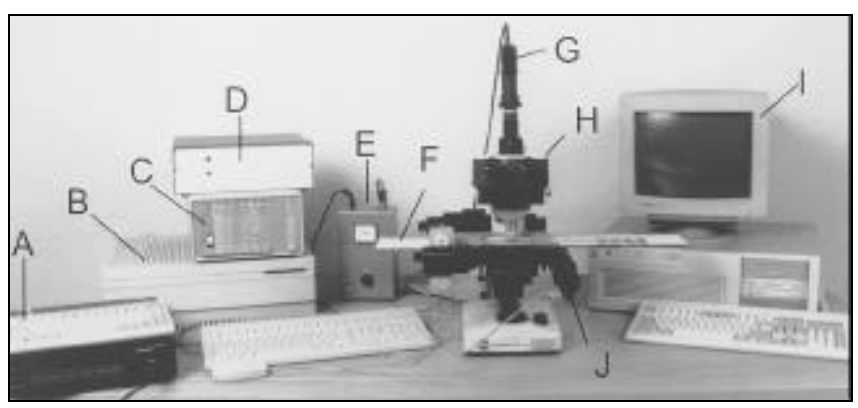

Figure 2. Overview of capture and delivery systems. (A) Optronics camera control unit, (B) Macintosh computer for video capture, (C) Ludl stage controller unit, (D) slide "jukebox" control unit, (E) light source control unit, (F) microscope 15-slide tray, (G) Optronics DEI-750 camera, (H) Leitz Laborlux 12 trinocular microscope, (I) Microsoft Windows NT computer and (J) Ludl $\mathrm{x}$-axis/y-axis motorized stage.

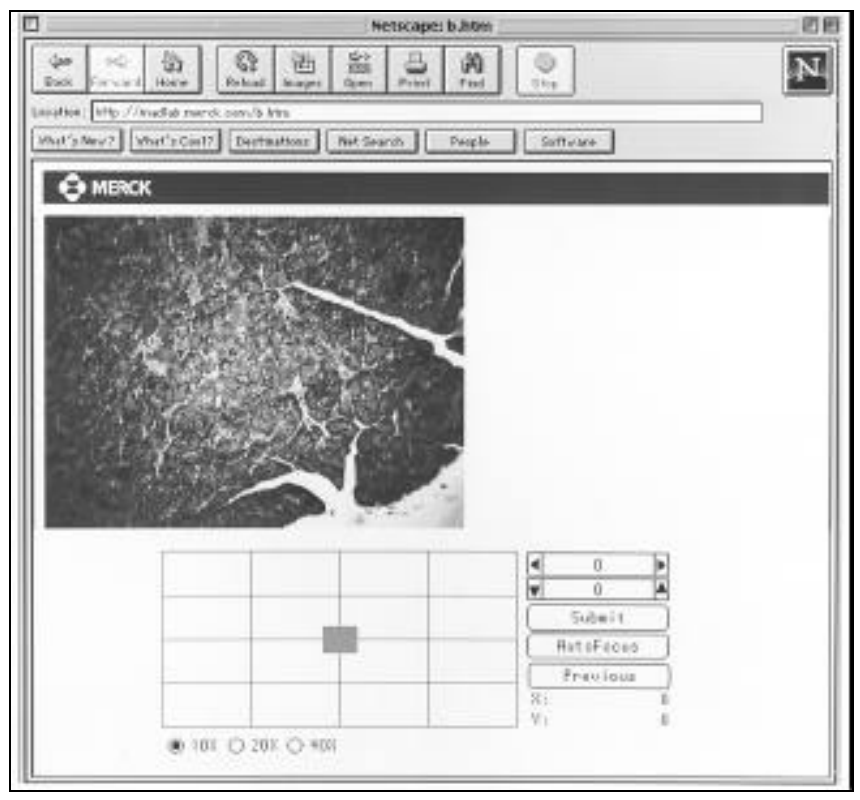

Figure 3. Screen capture of Phase II HTML page. The user has full control over the positioning of the microscope by a Shockwave movie. The user drags the center box to the desired position and clicks on the Submit button. As in Phase I, only the microscope image is refreshed. the stage, autofocus and objective scale selection (Figure 3). The gray grid area indicates the possible live area of the microscope slide, while the smaller blue box indicates the current area and location being viewed. To the right of the interactive grid are areas for the end user to enter either specific numerical values or increase/decrease in steps of 500 motor units (arbitrary units approximately equal to $100 \mu \mathrm{m}$ at $400 \times$ ). Currently, the system does not allow the user to mark the location for revisiting; however, the $\mathrm{x}$-axis, $\mathrm{y}$-axis coordinates are provided for notation. Below, there is a button to submit the values and begin the movement, along with an autofocus button and the coordinates from the previous position. The user can also select the magnification factor for the blue box. This was added as a future enhancement that will allow the user to change objectives as well.

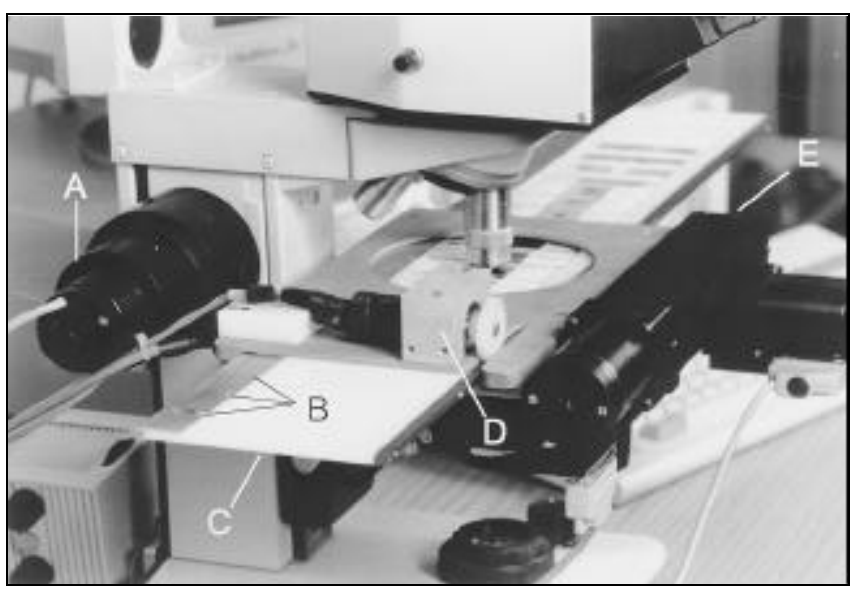

Figure 4. Custom-built slide holder. (A) Ludl autofocus controller motor (B) optical sensors, (C) 15-slide microscope tray, (D) spring-loaded, geared motor unit and (E) Ludl x-axis/y-axis motorized stage.

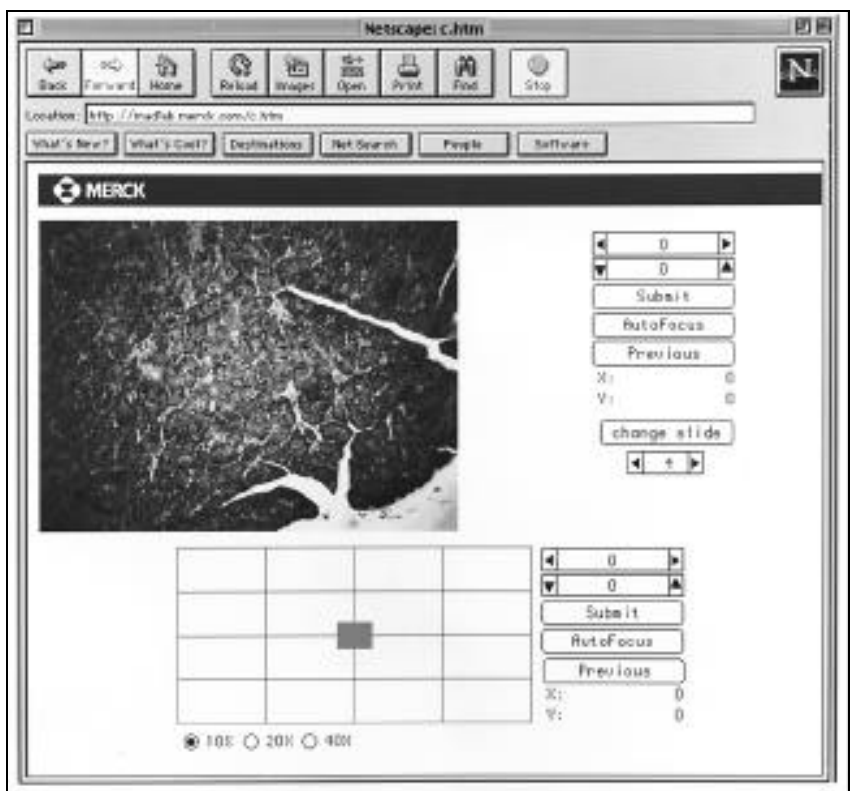

Figure 5. Screen capture of Phase III HTML page. An additional Shockwave movie was added to allow for the selection of one of 15 slides for viewing. Redundant controls were included for ease of use. 
Control of the $\mathrm{x}$-axis, $\mathrm{y}$-axis stage controller works as follows: $(i)$ the user moves the interactive box to the location of choice or manually enters $\mathrm{x}, \mathrm{y}$ coordinates; (ii) the user clicks on the submit button; (iii) the Shockwave movie submits the $\mathrm{x}$-axis and $\mathrm{y}$-axis coordinates back to the Purveyor server software in a form submission method; (iv) Purveyor then forwards the data to a custom-written common gateway interface (CGI); $(v)$ the CGI does the necessary mathematical calculations and then passes the data to NTperl; and (vi) NTperl recompiles the data and sends it out as an American Standard Code for Information Interchange (ASCII) text string to the Ludl controller. The Ludl reads the ASCII text and executes it, causing the stage to move. The time necessary to move from one side of the slide to another never took more than two refresh cycles to appear on the user's screen (maximum of a 14-s delay). While the custom CGI was being developed, the interface was also created. Using the Frame tags within Navigator, a screen was developed that used three rows: the first row showing the corporate logo, the second showing the image that refreshed by the Meta tag and the third showing the stage controller within the Shockwave movie (Figure 3).

\section{Phase III}

The final phase of the project required the design and fabrication of a custom slide loader capable of loading up to 15 different slides into the field of view of the microscope. The apparatus mounts to the $\mathrm{x}$-axis/y-axis stage of the microscope and positions slides under the objective through a multi-position linear slide carrier with integral gear rack, which is driven by a DC gear motor (Portescap, Hauppauge, NY, USA) and pinion gear. Optical sensors ensure proper homing and positioning of the slide carrier (Figure 4). The device is controlled by a Domino-52 Embedded Controller with a BASIC interpreter (Micromint, Vernon, CT, USA). The CGI directs simple ASCII commands over a serial port to the embedded controller, where a BASIC program interprets the commands, activates the drive motor and moves a slide into position. This stage allowed for the selection of one of 15 slides preset in the stage. This device is also controlled by a serial controller that uses the same CGI that controls the x-axis/y-axis stage. Using the Frame tags within Navigator, a screen was developed that used two rows and two columns. The first row showed the logo, the first column showed the micrograph, which was refreshed by the Meta tag, and the second column showed the Shockwave movie, which provided for the changing of the slide and exact positioning of the micrograph under the objective. The final row contained the second Shockwave movie, giving the user the same interface that they saw in Phase II (Figure 5). An additional limitation of a Phase III design is that the optical path cannot contain immersion optics, a feature available in Phases I and II.

\section{CONCLUSIONS}

There are several advantages to using this system: it allows for collaborative work to be done by researchers at different sites; it allows for immediate feedback on scientific issues; it allows for work to continue irrespective of time differences between sites; and it is easy to use. While not designed to replace larger, expensive, high-resolution telepathology systems currently in use over ISDN lines, this system can be a very cost-effective solution to allow a group of people to view the work from their own work areas, rather than one location, at any time of the day or night. Future enhancements include a migration to a more automated research microscope so that objectives can be changed by remote control; this is solely a function of the microscope platform used, as the programming features necessary to send the commands already exist. In addition, the image resolution will be improved upon. There is also the need for an on-demand digital image capture function. This would provide a high-resolution capture that can then be used for image analysis. In addition to on-demand capture, the authors are looking at providing an adjustable refresh rate, which would trade off resolution for speed when searching for an area of interest on a slide. The system is currently being upgraded to improve both features and performance. A ComputerEyes/1024 Video Capture Board (Digital Vision, Boston, MA, USA) is being added to the NT system. This board will eliminate the need for the Macintosh computer, which is currently handling the capture function. Along with the video board, two pieces of software, Fsx Server and EmuLive, (JcS Canada, Montreal, QC, Canada) are being added, which will allow for the display of live video at a rate of six frames per second. Finally, since this is a collaborative research tool that provides images, an area will be set up for the posting of images with room for others to post comments and discussions.

\section{ACKNOWLEDGMENTS}

The authors would like to acknowledge the help of Spatz Computer Graphics (Rivers Edge, NJ, USA) for their help with the Perl scripting, Chris Hanas and Kevin Blake of the Bioelectronics Department and Jill Baiml of the Visual Communications Department at Merck Research Laboratories for their engineering support for the Phase III stage and for the photographs, respectively.

\section{REFERENCES}

1.Bashshur, R. 1995. On the definition and evaluation of telemedicine. Telemedicine 1:19-30.

2.Chumbley, L.S., M. Meyer, K. Fredrickson and F. Laabs. 1995. Computer networked scanning microscope for teaching, research, and industry applications. Microscopy Res. and Techn. 32:330-336.

3.Waddel, M. Real time remote microscope control over the Internet. Tucson: University of Arizona; 1992. Thesis.

Received 26 February 1997; accepted 21 April 1997.

Address correspondence to:

Ron Maturo

Merck \& Co.

RY80M-265

P.O. Box 2000

Rahway, NJ 07065, USA

Internet:maturo@merck.com 